\title{
Overexpression of fibulin- 4 is associated with tumor progression and poor prognosis in patients with cervical carcinoma
}

\author{
JIE CHEN $^{1}$, JIE ZHANG $^{2}$, XI LIU $^{3}$, RUI FANG $^{3}$, YUERAN ZHAO $^{2}$ and DEMEI MA ${ }^{4}$ \\ ${ }^{1}$ Department of Maternal and Child Health Care, School of Public Health, Shandong University, Jinan, Shandong 250012; \\ ${ }^{2}$ Central Laboratory, Provincial Hospital Affiliated to Shandong University, Jinan, Shandong 250021; \\ ${ }^{3}$ Clinical Medicine, School of Medicine, Shandong University, Jinan, Shandong 250012; ${ }^{4}$ Department of Obstetrics \\ and Gynecology, Second Hospital of Shandong University, Jinan, Shandong 250033, P.R. China
}

Received February 14, 2014; Accepted April 3, 2014

DOI: $10.3892 /$ or.2014.3139

\begin{abstract}
Fibulin-4, a member of the fibulin family of extracellular glycoproteins, is implicated in the progression of a number of types of cancer. However, the function of fibulin-4 in cervical cancer progression remains unexplored. Fibulin-4 mRNA and protein expression levels in normal cervical tissue, cervical intraepithelial neoplasia (CIN), cervical carcinoma, highly invasive subclones and low-invasive subclones were evaluated by real-time reverse transcriptase-polymerase chain reaction and immunohistochemistry. Serum fibulin-4 levels in patients with CIN and cervical carcinoma were measured by enzyme-linked immunosorbent assay. To assess the angiogenic properties of fibulin-4, vascular endothelial growth factor (VEGF) expression and tumor microvessel density (MVD) were analyzed in the cervical carcinoma cases by immunohistochemistry. Fibulin-4 expression was upregulated in the cervical carcinoma cases, and was positively correlated with MVD and VEGF expression. Fibulin-4 overexpression and high serum levels were significantly associated with advanced stage, low differentiation, lymph node metastasis, and poor prognosis in patients with cervical cancer. Fibulin-4 expression was also found to be overexpressed in highly invasive subclones when compared with the low-invasive subclones. Fibulin-4 is a newly identified glycoprotein that is overexpressed in cervical carcinoma. Fibulin-4 promotes angiogenesis and is associated with poor prognostic clinicopathologic features This study demonstrated that fibulin-4 may serve as a new prognostic factor and as a potential therapeutic target for patients with cervical carcinoma.
\end{abstract}

Correspondence to: Dr Demei Ma, Department of Obstetrics and Gynecology, Second Hospital of Shandong University, Jinan, Shandong 250033, P.R. China

E-mail: mademei2013@163.com

Key words: fibulin-4, cervical carcinoma, angiogenesis, prognosis

\section{Introduction}

In women, cervical cancer is the second most common cancer in developing regions with more than $85 \%$ of the global burden occurring in developing countries (1). Although cervical cancer screening has reduced its incidence, $\sim 30 \%$ of patients are still diagnosed at an advanced stage and ultimately show recurrence and metastasis (2). Therefore, investigation of the mechanisms of tumor invasion and metastasis will provide further insight into the occurrence and development of cervical cancer. In recent years, many genes such as secreted protein acidic and rich in cysteine (3), metastasis-associated 1 (4), and twist homolog 2 (5) have been found to be correlated with the progression of cervical cancer. However, few studies have explored the relationship between fibulin-4 and cervical cancer progression and prognosis.

Fibulin-4, also known as endothelial growth factor (EGF)-containing fibulin-like extracellular matrix protein 2 (EFEMP2), mutant p53 binding protein 1 (MBP1) or UPH1, is a 443-amino acid secreted protein that contains six EGF-like calcium-binding domains and belongs to the fibulin family (6). Fibulins have been shown to modulate cell morphology, growth, adhesion and motility, and are closely associated with the development of a wide variety of carcinomas (7). As tumor-suppressor genes, fibulin-2 $(8,9)$ and fibulin-5 (10-12) were widely considered to be associated with the suppression of tumor growth, invasion and angiogenesis. Research findings on the role of fibulin-1 and fibulin-3 in different tumor tissues have been controversial. Few researchers have reported oncogenic activities (13-19), whereas others have reported tumor-suppressive activities (20-27). This discrepancy may be attributable to the influence of the tumor microenvironment on tumor-associated genes in promoting angiogenesis and metastasis (28).

Fibulin-4 is essential for connective tissue development and elastic fiber formation and may also play an important role in vascular patterning and collagen biosynthesis (29). Fibulin-4 plays a role in many clinical conditions such as cutis laxa (30), aortic aneurysms (31), osteoarthritis (32) and cancer $(22,33)$. In their study on colon tumors, Gallagher et al found that the fibulin-4 gene was localized on chromosome 11q13 (33); translocations, amplifications and other rearrangements in this 
region are associated with a variety of human cancers $(34,35)$. Reverse transcriptase (RT)-polymerase chain reaction (PCR) of RNA from paired human colon tumors and adjacent normal tissue showed that tumors had a 2-7 fold increase in the level of fibulin-4 mRNA expression (33). However, in prostate cancer (22), fibulin-4 was found to be significantly downregulated and weakly expressed in carcinoma cell lines compared to normal prostate epithelial cells. Against this background of controversies in the research addressing the role of fibulin-4, more studies are needed to elucidate the relationship between fibulin- 4 and cancer. To our knowledge, the role of fibulin- 4 in cervical cancer remains unexplored.

The purpose of this study was to assess whether fibulin-4 expression is associated with the progression of cervical cancer and to investigate the relationship between fibulin-4 and angiogenesis.

\section{Materials and methods}

Cell lines. Highly invasive subclones (HeLa-1 and SiHa-1) and low-invasive subclones (HeLa-25 and $\mathrm{SiHa}-23$ ) were derived from the HeLa and SiHa human cervical cancer cell lines, using the limited dilution method. Next, the cell electrophoretic mobility (EPM) of each clone was measured to study the charge-related properties using microcapillary electrophoresis chips. Finally, the MTT assay, soft agar colony formation assay, Matrigel invasion assay and cell migration assay were performed and tumor xenografts were generated in nude mice to confirm that highly invasive subclones and low-invasive subclones had high and low metastatic potential, respectively (3). Cells were cultured in Dulbecco's modified Eagle's medium (DMEM) supplemented with $10 \%$ fetal bovine serum (FBS) and antibiotics (Gibco-BRL, Rockville, MD, USA).

Tissue specimens. A total of 270 human cervical tissue specimens obtained with written informed consent from patients were used for this study. Two hundred and thirty cervical cancer patients were enrolled from the Department of Gynecology and Obstetrics, Shandong Provincial Hospital between 2006 and 2010. There were 60 cervical intraepithelial neoplasia (CIN) cases [age range, 28-55 years; mean (SD), 40 (8)], 140 squamous cell carcinoma cases [age range, 30-65 years; mean (SD), 45 (10)] and 30 adenocarcinoma cases [age range, 35-60; mean (SD), 43 (6)]. All cervical cancer patients were clinically staged according to the revised International Federation of Gynecology and Obstetrics (FIGO) staging system (FIGO stage I, 76 cases; FIGO stage II, 81 cases; and FIGO stage III and IV, 13 cases). None of the cervical cancer patients received preoperative radiation or chemotherapy. All patients were treated consecutively and were followed up regularly; 9 patients were lost to follow-up and 25 patients died during the study period. Follow-up duration was between 2 and 7 years by the end of 2012. Forty normal cervical tissue specimens [age range, 30-60 years; mean (SD), 47 (9)] were obtained from the Department of Gynecology and Obstetrics, Shandong Provincial Hospital. The study was approved by the Institutional Medical Ethics Committee of Shandong University.

Blood samples. Blood samples were obtained with written informed consent from the same 230 cervical cancer patients
(60 CIN cases, 140 squamous cell carcinoma cases and 30 adenocarcinoma cases) at the Department of Gynecology and Obstetrics, Shandong Provincial Hospital between 2006 and 2010. None of the cervical cancer patients received preoperative radiation or chemotherapy. Forty control blood samples were obtained with written informed consent from age-matched examinees undergoing health examinations at Shandong Provincial Hospital. Control subjects had no history of disease and no abnormalities on laboratory examinations. The study was approved by the Institutional Medical Ethics Committee of Shandong University.

Enzyme-linked immunosorbent assay. Levels of fibulin-4 in serum samples were measured using sandwich enzyme-linked immunosorbent assay (ELISA) with human fibulin-4 ELISA assay kits (Immuno-Biological Laboratories, Fujioka, Gunma, Japan). Serum was diluted with enzyme immunoassay (EIA) buffer ( $1 \%$ bovine serum albumin, $0.05 \%$ Tween-20 in phosphate buffer) and incubated for $2 \mathrm{~h}$ at $37^{\circ} \mathrm{C}$. After 4 washes with EIA buffer, horseradish peroxidase-conjugated antibodies were added and incubated for $30 \mathrm{~min}$ at $4^{\circ} \mathrm{C}$. After 4 washes, $100 \mu \mathrm{l}$ of tetramethylbenzidine solution was added and incubated for $30 \mathrm{~min}$ at room temperature. The reaction was stopped with $100 \mu \mathrm{l}$ of $1 \mathrm{~N}$ sulfuric acid and measured using the ELISA reader at $450 \mathrm{~nm}$.

Immunohistochemistry. According to standard streptavidinbiotin-peroxidase complex procedures, immunohistochemistry (IHC) was performed on formalin-fixed, paraffin-embedded sections $(5-\mu \mathrm{m}$ thick) and cell slides were fixed in $4 \%$ paraformaldehyde. Briefly, after dewaxing, rehydration, and antigen retrieval, the sections were incubated with anti-human fibulin-4 antibodies (ab125073; Abcam and MAB2644; Millipore) with working dilutions of 1:200 for ab125073 and 1:500 for MAB2644 at $4^{\circ} \mathrm{C}$ overnight, and stained with the enzyme substrate 3',3-diaminobenzidine tetrahydrochloride (Sigma, St. Louis, MO, USA). Human breast cancer paraffinembedded sections (fibulin-4-positive) were used as positive controls. A negative control was obtained by replacing the primary antibody with normal rabbit or mouse immunoglobulin (IgG). Positive expression of fibulin-4 protein was defined as the presence of brown granules in the cytoplasm.

Immunohistochemistry analysis. A semi-quantitative scoring system derived from the method by Soumaoro et al (36) for both the intensity of staining and the percentage of positive cells was used to evaluate fibulin-4 expression. The intensity of fibulin-4-positive staining was scored from 0 to 3 (negative, 0 ; weak, 1; moderate, 2; or strong, 3), and the percentage of positively stained cells was scored as $0(0 \%), 1(1-25 \%), 2$ $(26-50 \%), 3(51-75 \%)$ and $4(76-100 \%)$. The sum of the intensity and percentage scores was used as the final staining score $(0-7)$. The sum-indices $(-),(+),(++)$, and $(+++)$ indicated final staining scores of 0, 1-3, 4-5 and 6-7, respectively. For statistical analysis, sum-indices (-) and (+) were defined as low fibulin-4 expression, while sum-indices $(++)$ and $(+++)$ were defined as high fibulin-4 expression. Each section was independently scored by two pathologists. In cases of an inconsistency, a third pathologist was consulted to arrive at a consensus. To assess reproducibility, we invited three other pathologists to 

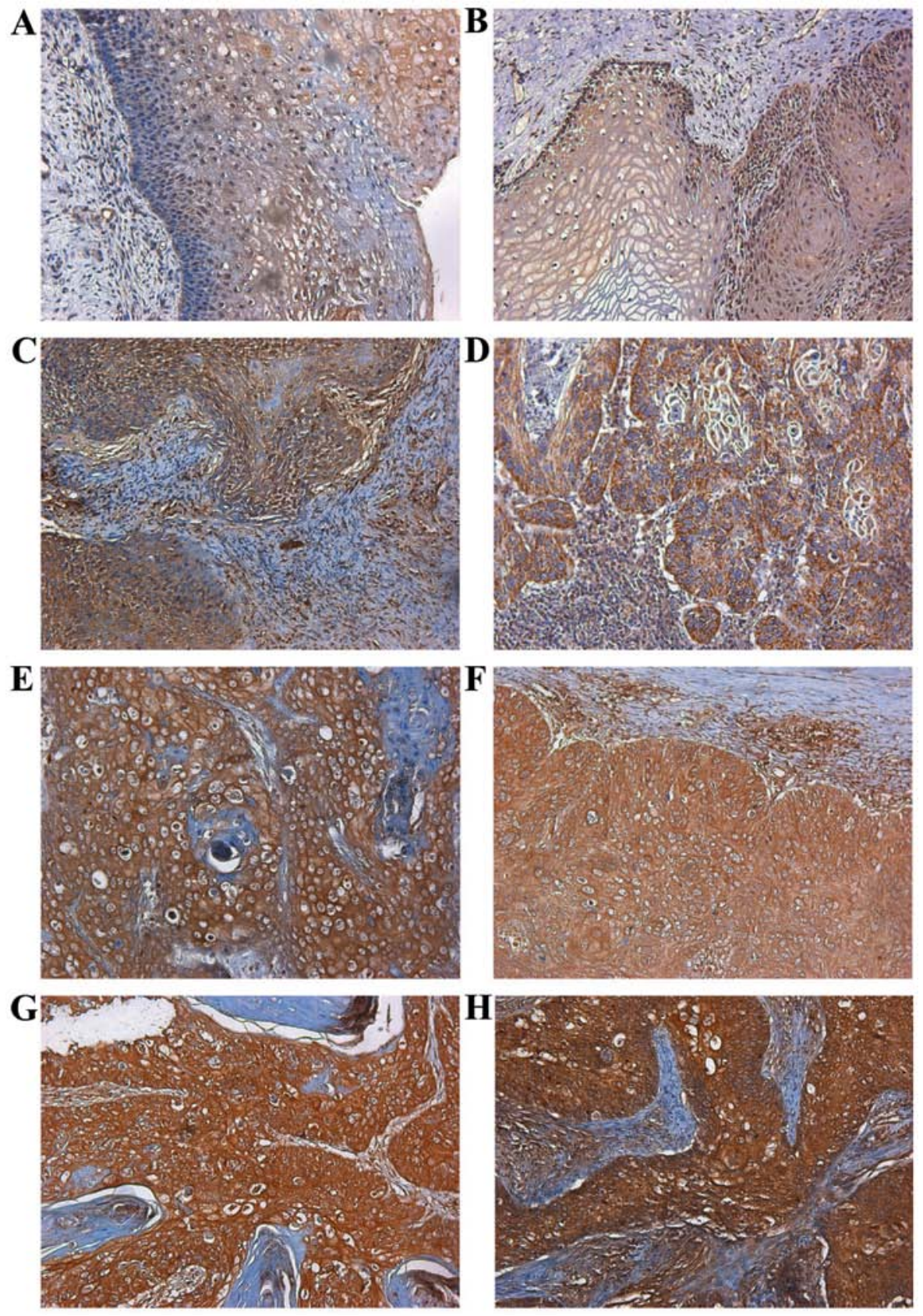

Figure 1. Expression of fibulin-4 in human cervical tissues with the ab125073 antibody. (A) Normal human cervical tissue, (B) cervical intraepithelial neoplasia (CIN), (C and D) stage I cervical carcinoma, (E and F) stage II cervical carcinoma, (G and H) stage III and IV cervical carcinoma (magnification, x200).

score all sections independently. The interobserver reliability and intraobserver reproducibility of IHC experiments were evaluated using kappa $(\kappa)$ statistical evaluation.

Microvessel assessment. Microvessel density (MVD) was assessed according to CD34 immunohistochemical staining of tumor vessels. Any immune-positive single endothelial cell or endothelial cell clusters and microvessels in the tumor were considered to be individual vessels and were counted, as described by Weidner et al (37). Peritumoral vascularity, vascularity in areas of necrosis, and vessels with a thick muscle wall or having a diameter larger than 8 erythrocytes, were not counted. The sections were scanned at low power $(\mathrm{x} 100)$ to select the most vascularized (hot-spots) areas. The microvessels in the hot-spots were then counted, and an average count in three hot spots was calculated as the MVD. All counts were performed independently by three observers who were blinded to the corresponding clinicopathological data.

Quantitative real-time-polymerase chain reaction. Total RNA was extracted using TRIzol reagent (Invitrogen) and reverse transcribed. Quantitative real-time RT-PCR analysis was performed using ABI Prism 7500 Real-Time PCR System (Applied Biosystems). Each well (20- $\mu 1$ reaction volume) contained $10 \mu 1$ Power SYBR-Green PCR Master Mix (Applied Biosystems), $1 \mu \mathrm{l}$ of each primer ( $5 \mu \mathrm{mol} / \mathrm{l})$ and $1 \mu \mathrm{l}$ template. The following primers were used: fibulin- 4,5 '-GCTGCTACT GTTGCTCTTGGG-3' and 5'-GGGATGGTCAGACACTCGT TG-3'; $\beta$-actin 5'-CCACGAAACTACCTTCAACTCCA-3' and 5'-GTGATCTCCTTCTGCATCCTGTC-3'. 

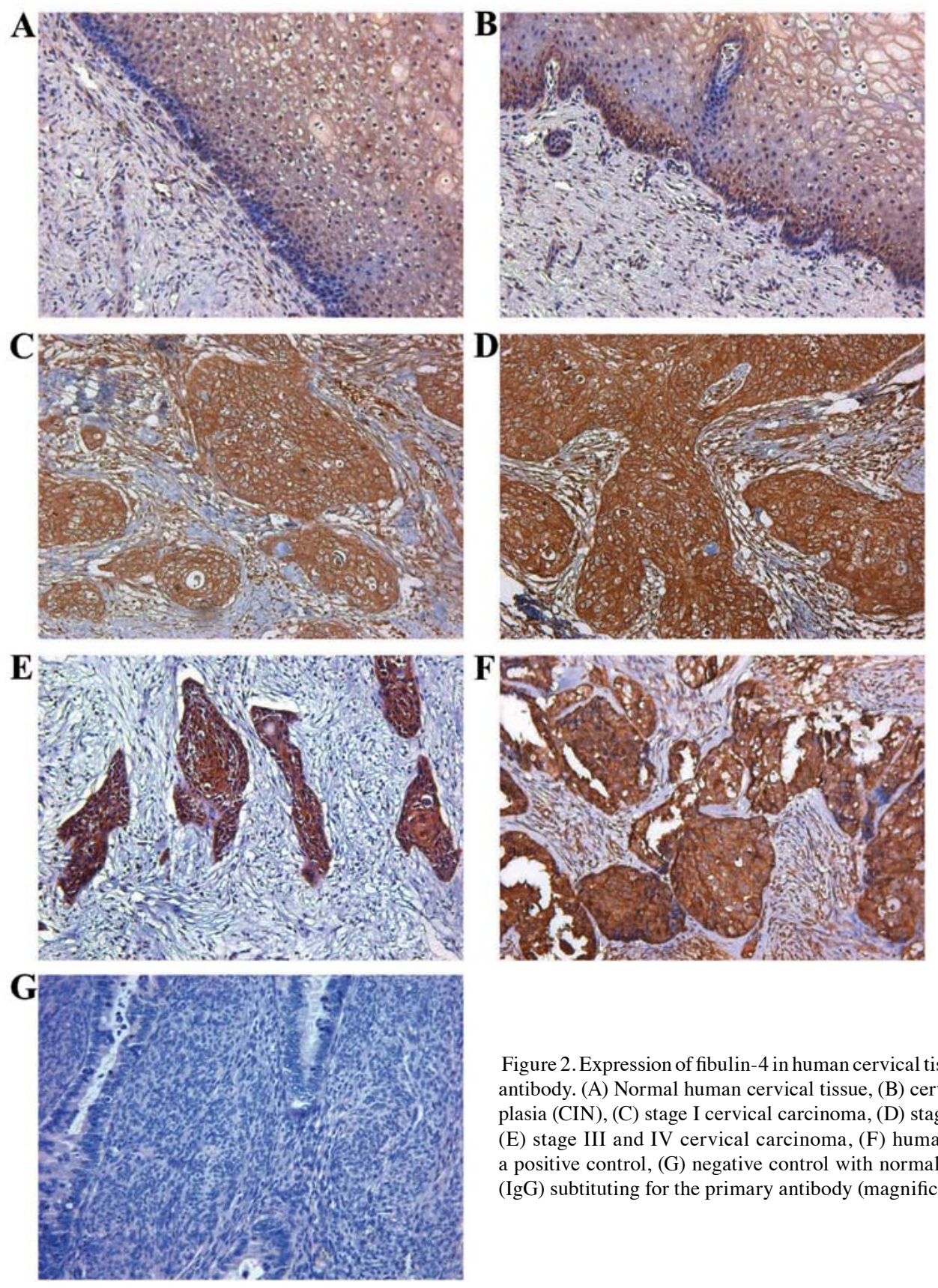

Figure 2. Expression of fibulin- 4 in human cervical tissues with the MAB2644 antibody. (A) Normal human cervical tissue, (B) cervical intraepithelial neoplasia (CIN), (C) stage I cervical carcinoma, (D) stage II cervical carcinoma, (E) stage III and IV cervical carcinoma, (F) human breast cancer used as a positive control, (G) negative control with normal rabbit immunoglobulin (IgG) subtituting for the primary antibody (magnification, $\mathrm{x} 200$ ).

Statistical analysis. IHC data were analyzed using the Chi-square test. Measurement data were expressed as means \pm SE. The interobserver reliability and intraobserver reproducibility of IHC experiments were evaluated using the $\kappa$ statistical evaluation. The strength of agreement was interpreted as follows: excellent $(\kappa \geq 0.80)$, good $(0.60-0.79)$, moderate $(0.40-0.59)$, poor $(0.20-0.39)$ and very poor $(<0.20)$ (38). For comparison of means between two groups, a two-tailed t-test was used and for comparison of means among three groups, one-way ANOVA was used. Survival curves were calculated using the Kaplan-Meier method and analyzed using the log-rank test. Correlations of fibulin-4 expression with VEGF expression and MVD were analyzed using the Pearson correlation test. Multivariate Cox proportional hazards models were used to define the potential prognostic significance of individual parameters. Statistical analysis

was performed using SPSS software version 13.0. Two-sided P-values of $<0.05$ were considered to indicate statistically significant differences.

\section{Results}

Fibulin-4 expression in the human cervical tissues. Fibulin-4 protein expression was extremely low in the normal human cervical tissue and CIN (Figs. 1A and B; 2A and B). However, in most cervical carcinomas, fibulin-4 immunoreactivity was high, and high fibulin- 4 protein expression was detected in the cytoplasm of cervical cancer cells (Figs. 1C-H; 2C-E). Moreover, high fibulin-4 protein expression was associated with low differentiation, advanced stage and positive lymph node status of the cervical carcinoma cases (Tables I and II). The interobserver reliability coefficients were 0.86 and 0.81 
Table I. Protein expression of fibulin-4 in the human cervical tissues with the ab125073 antibody.

\begin{tabular}{|c|c|c|c|c|c|c|c|}
\hline & \multirow[b]{2}{*}{$\mathrm{N}$} & \multicolumn{2}{|c|}{$\begin{array}{l}\text { Fibulin-4 low } \\
\qquad(-/+)\end{array}$} & \multicolumn{2}{|c|}{$\begin{array}{l}\text { Fibulin-4 high } \\
\qquad(++/+++)\end{array}$} & \multirow[b]{2}{*}{$\chi^{2}$} & \multirow[b]{2}{*}{ P-value } \\
\hline & & $\mathrm{n}$ & $(\%)$ & $\mathrm{n}$ & $(\%)$ & & \\
\hline Normal & 40 & 37 & $(92.5)$ & 3 & $(7.5)$ & 66.05 & $<0.01$ \\
\hline CIN & 60 & 44 & $(73.3)$ & 16 & $(26.7)$ & & \\
\hline Carcinoma & 170 & 53 & $(31.2)$ & 117 & $(68.8)$ & & \\
\hline Pathological type & & & & & & 0.02 & $>0.05$ \\
\hline Squamous cell carcinoma & 140 & 44 & $(31.4)$ & 96 & $(68.6)$ & & \\
\hline Adenocarcinoma & 30 & 9 & $(30.0)$ & 21 & $(70.0)$ & & \\
\hline Cell differentiation & & & & & & 25.57 & $<0.01$ \\
\hline High and medium & 89 & 43 & $(48.3)$ & 46 & $(51.7)$ & & \\
\hline Low & 81 & 10 & $(12.3)$ & 71 & $(87.7)$ & & \\
\hline Tumor stage & & & & & & 26.07 & $<0.01$ \\
\hline I & 60 & 32 & $(53.3)$ & 28 & $(46.7)$ & & \\
\hline II & 59 & 19 & $(32.2)$ & 40 & $(67.8)$ & & \\
\hline III and IV & 51 & 4 & $(7.8)$ & 47 & $(92.2)$ & & \\
\hline Nodal status & & & & & & 18.26 & $<0.01$ \\
\hline Positive & 66 & 8 & (12.1) & 58 & (87.9) & & \\
\hline Negative & 104 & 45 & (43.3) & 59 & $(56.7)$ & & \\
\hline
\end{tabular}

CIN, cervical intraepithelial neoplasia.

Table II. Protein expression of fibulin-4 in the human cervical tissues with the MAB2644 antibody.

\begin{tabular}{|c|c|c|c|c|c|c|c|}
\hline & \multirow[b]{2}{*}{$\mathrm{N}$} & \multicolumn{2}{|c|}{$\begin{array}{l}\text { Fibulin-4 low } \\
\qquad(-/+)\end{array}$} & \multicolumn{2}{|c|}{$\begin{array}{l}\text { Fibulin-4 high } \\
\qquad(++/+++)\end{array}$} & \multirow[b]{2}{*}{$\chi^{2}$} & \multirow[b]{2}{*}{ P-value } \\
\hline & & $\mathrm{n}$ & $(\%)$ & $\mathrm{n}$ & $(\%)$ & & \\
\hline Normal & 40 & 36 & $(90)$ & 4 & $(10)$ & 63.468 & $<0.01$ \\
\hline CIN & 60 & 42 & $(70)$ & 18 & $(30)$ & & \\
\hline Carcinoma & 170 & 50 & $(29.4)$ & 120 & $(70.6)$ & & \\
\hline Pathological type & & & & & & 0.270 & $>0.05$ \\
\hline Squamous cell carcinoma & 140 & 40 & $(28.6)$ & 100 & $(71.4)$ & & \\
\hline Adenocarcinoma & 30 & 10 & $(33.3)$ & 20 & $(66.7)$ & & \\
\hline Cell differentiation & & & & & & 21.705 & $<0.01$ \\
\hline High and medium & 89 & 40 & $(44.9)$ & 49 & $(55.1)$ & & \\
\hline Low & 81 & 10 & $(12.3)$ & 71 & $(87.7)$ & & \\
\hline Tumor stage & & & & & & 32.759 & $<0.01$ \\
\hline I & 76 & 39 & $(51.3)$ & 37 & $(48.7)$ & & \\
\hline II & 81 & 11 & $(13.6)$ & 70 & $(86.4)$ & & \\
\hline III and IV & 13 & 0 & (0) & 13 & $(100)$ & & \\
\hline Nodal status & & & & & & 24.525 & $<0.01$ \\
\hline Positive & 66 & 6 & $(9.1)$ & 60 & $(90.9)$ & & \\
\hline Negative & 104 & 47 & $(45.2)$ & 57 & $(54.8)$ & & \\
\hline
\end{tabular}

CIN, cervical intraepithelial neoplasia.

for the first and second assessments, with an intraobserver reproducibility coefficient of 0.85 . The interobserver reliability and intraobserver reproducibility of the IHC experiments were excellent. Similarly results were also found for the real-time 
Table III. mRNA expression of fibulin-4 in the human cervical tissues.

\begin{tabular}{|c|c|c|c|}
\hline & $\mathrm{N}$ & Fibulin-4 mRNA & P-value \\
\hline Control & 40 & $0.0096 \pm 0.0064$ & \\
\hline CIN & 60 & $0.0091 \pm 0.0048$ & $>0.05^{\mathrm{a}}$ \\
\hline Carcinoma & 170 & $0.0769 \pm 0.0089$ & $<0.05^{\mathrm{b}}$ \\
\hline \multicolumn{3}{|l|}{ Pathological type } & \multirow[t]{3}{*}{$>0.05$} \\
\hline $\begin{array}{l}\text { Squamous cell } \\
\text { carcinoma }\end{array}$ & 140 & $0.0648 \pm 0.0115$ & \\
\hline Adenocarcinoma & 30 & $0.0796 \pm 0.0127$ & \\
\hline \multicolumn{3}{|l|}{ Cell differentiation } & \multirow[t]{3}{*}{$<0.05$} \\
\hline High and medium & 89 & $0.0284 \pm 0.0078$ & \\
\hline Low & 81 & $0.0932 \pm 0.0105$ & \\
\hline \multicolumn{3}{|l|}{ Tumor stage } & \multirow[t]{4}{*}{$<0.05$} \\
\hline I & 76 & $0.0267 \pm 0.0073$ & \\
\hline II & 81 & $0.0541 \pm 0.0081$ & \\
\hline III and IV & 13 & $0.0946 \pm 0.0126$ & \\
\hline \multicolumn{3}{|l|}{ Nodal status } & \multirow[t]{3}{*}{$<0.05$} \\
\hline Positive & 66 & $0.0979 \pm 0.0117$ & \\
\hline Negative & 104 & $0.0243 \pm 0.0059$ & \\
\hline \multicolumn{4}{|c|}{$\begin{array}{l}{ }^{a} \mathrm{CIN} \text { compared with healthy control, } \mathrm{P}>0.05 \text {; }{ }^{\mathrm{b}} \text { cervical carcinoma } \\
\text { compared with healthy control and } \mathrm{CIN}, \mathrm{P}<0.05 \text {. CIN, cervical } \\
\text { intraepithelial neoplasia. }\end{array}$} \\
\hline
\end{tabular}

Table IV. Predictive factors of survival by multivariate analysis (Cox proportional hazards model).

\begin{tabular}{lcc}
\hline Prognostic factors & HR $(95 \% \mathrm{CI})$ & P-value \\
\hline Fibulin-4 & $1.019(1.007-1.031)$ & 0.002 \\
Pathological type & $0.978(0.429-2.230)$ & 0.957 \\
Cell differentiation & $1.012(0.999-1.026)$ & 0.065 \\
Tumor stage & $3.175(1.361-7.403)$ & 0.007 \\
Lymph node metastasis & $2.129(1.319-3.435)$ & 0.002 \\
Tumor size & $1.095(0.986-1.216)$ & 0.089 \\
Age (years) & $0.981(0.957-1.005)$ & 0.125 \\
\hline
\end{tabular}

HR, hazard ratio; CI, confidence interval.

RT-PCR experiment; fibulin-4 mRNA expression was also extremely low in the normal cervical tissues and CIN, and significantly high fibulin- 4 expression was noted in the cervical carcinoma cases. Moreover, high fibulin-4 mRNA expression was also associated with low differentiation, advanced stage and positive lymph node status of the cervical carcinomas (Table III). To evaluate the prognostic value of fibulin-4 in cervical cancer, we performed survival analysis using KaplanMeier analysis. The results showed that patients with high fibulin-4 expression had a much worse prognosis than those with low fibulin-4 expression (log-rank, P<0.01) (Fig. 3A). In the multivariate analysis, considering all histological and
A
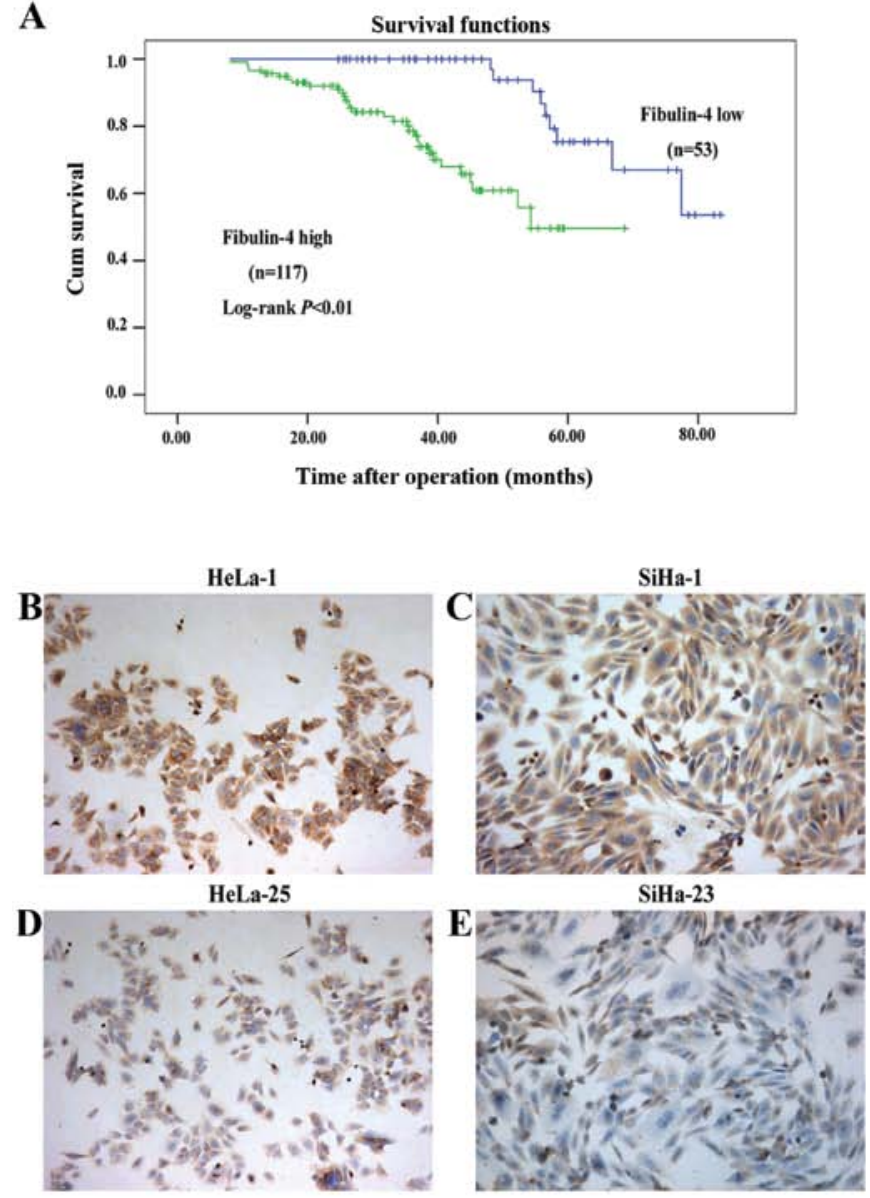

$\mathbf{F}$

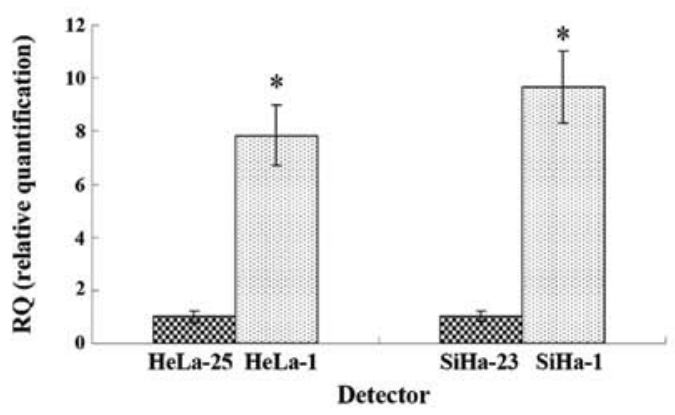

Figure 3. Kaplan-Meier analysis and fibulin-4 expression in the highly invasive subclones and in the low-invasive subclones. (A) Kaplan-Meier analysis of the overall survival of the patients whose tumors had high or low fibulin-4 expression. (B-E) Fibulin-4 protein expression in the highly invasive subclones (B) HeLa-1 and (C) SiHa-1 and low-invasive subclones (D) HeLa-25 and (E) SiHa-23 as measured by IHC staining (magnification, x200). (F) Fibulin-4 mRNA expression in the highly invasive subclones HeLa-1 and SiHa-1 and low-invasive subclones HeLa-25 and SiHa-23 as measured by qRT-PCR. "P<0.05 vs. control.

molecular features together, the significant prognostic factors were lymph node metastasis $(\mathrm{P}=0.002$; hazard ratio 2.129$)$, fibulin-4 expression ( $\mathrm{P}=0.002$; hazard ratio 1.019) and tumor stage $(\mathrm{P}=0.007$; hazard ratio 3.175$)$ (Table IV).

Differential expression of fibulin-4 in the highly invasive subclones and the low-invasive subclones. The highly invasive subclones (HeLa-1 and SiHa-1) and the low-invasive subclones (HeLa-25 and SiHa-23) were derived from the HeLa 

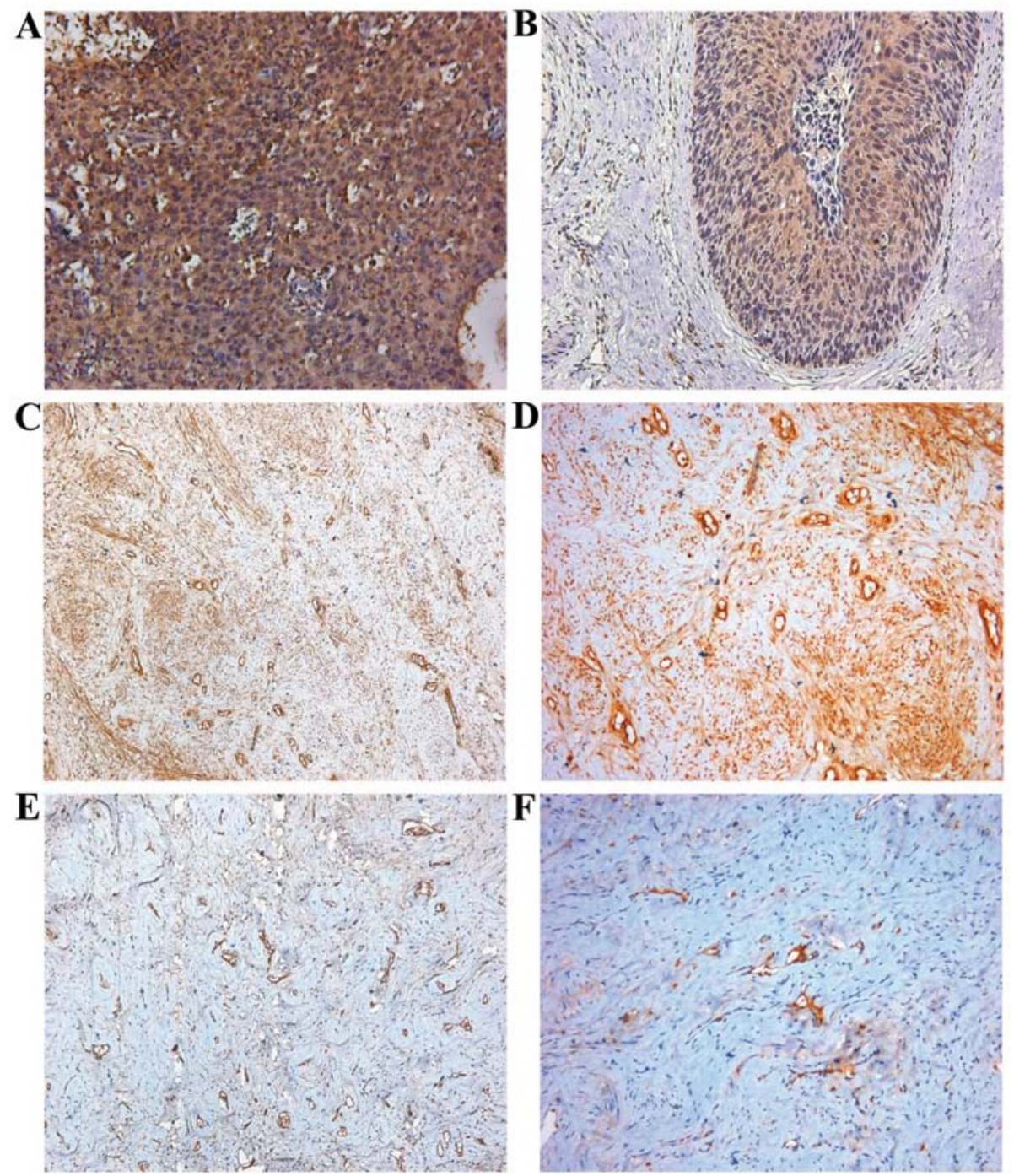

Figure 4. Immunohistochemical staining of VEGF and CD34 for determination of microvessel density (MVD). Immunohistochemical staining of VEGF in stages IV cervical carcinoma (A), and stage I cervical carcinoma (B) (magnification, x200). Immunohistochemical staining of CD34 for MVD in stage IV cervical carcinoma (C, x100 magnification) (D, x200 magnification), and in stage I cervical carcinoma (E, x100 magnification) (F, x200 magnification).

and SiHa human cervical cancer cell lines, using the limited dilution method. Since the cell lines have similar genetic backgrounds, they are suitable for comparative analysis. As shown in Fig. 3B-F, fibulin-4 protein and mRNA expression levels were extremely high in the highly invasive subclones (HeLa-1 and SiHa-1), compared to the low-invasive subclones (HeLa-25 and SiHa-23).

Serum levels of fibulin-4 in human cervical cancer patients and healthy control. As shown in Table V, the serum fibulin-4 level in cervical carcinoma patients was much higher than that in the healthy controls and CIN patients $(\mathrm{P}<0.05)$. No significant difference was found between healthy controls and CIN ( $>0.05)$. Moreover, high serum levels of fibulin-4 were associated with low differentiation, advanced stage and positive lymph node status of the cervical carcinoma cases $(\mathrm{P}<0.05)$. There were no significant differences among the different pathological types of cervical carcinoma $(\mathrm{P}>0.05)$.

Relationships of fibulin-4 with VEGF expression and MVD. Fig. 4 shows the representative immunohistochemical staining images for VEGF and CD34. The immunohistochemical expression of VEGF and fibulin-4 was evaluated using software Image-Pro Plus 6.0 to detect photodensity. In brief, five positive fields in a section were selected at random and then read using Image-Pro Plus 6.0, and the average densities were then calculated. Pearson correlation tests of MVD (Fig. 5A, $\mathrm{P}<0.01$ ) and VEGF expression (Fig. 5B, $\mathrm{P}<0.01$ ) vs. fibulin-4 revealed strong positive correlations.

\section{Discussion}

In the present study, we demonstrated for the first time that the expression of fibulin-4 is associated with poor prognostic clinicopathologic features, neovascularization and poor outcome in human cervical carcinoma patients.

Our immunohistochemical studies showed an upregulation of fibulin-4 expression in cervical carcinoma tissues, compared with normal cervical tissues and CIN. Fibulin- 4 is rich in elastic fiber tissue, and is mainly involved in the synthesis and arrangement of elastic fibers (39). In the present study, the expression of fibulin-4 in the extracellular matrix was found 
Table V. Serum levels of fibulin-4 in the patients with cervical tumors.

\begin{tabular}{lrcc}
\hline & N & Fibulin-4 (ng/ml) & P-value \\
\hline Control & 40 & $108.43 \pm 11.86$ & \\
CIN & 60 & $116.57 \pm 13.24$ & $>0.05^{\text {a }}$ \\
Carcinoma & 170 & $356.49 \pm 22.15$ & $<0.05^{\text {b }}$ \\
Pathological type & & & $>0.05$ \\
$\quad \begin{array}{l}\text { Squamous cell } \\
\text { carcinoma }\end{array}$ & 140 & $267.26 \pm 16.54$ & \\
$\quad$ Adenocarcinoma & 30 & $271.32 \pm 18.61$ & \\
Cell differentiation & & & $<0.05$ \\
$\quad$ High and medium & 89 & $106.93 \pm 10.22$ & \\
$\quad$ Low & 81 & $347.56 \pm 23.47$ & \\
Tumor stage & & & $<0.05$ \\
I & 76 & $109.84 \pm 13.51$ & \\
II & 81 & $213.47 \pm 15.69$ & \\
$\quad$ III and IV & 13 & $368.51 \pm 24.85$ & \\
Nodal status & & & $<0.05$ \\
$\quad$ Positive & 66 & $357.34 \pm 20.06$ & \\
$\quad$ Negative & 104 & $113.92 \pm 13.43$ & \\
\hline
\end{tabular}

${ }^{\mathrm{a}} \mathrm{CIN}$ compared with healthy control, $\mathrm{P}>0.05$; ${ }^{\mathrm{b}}$ cervical carcinoma compared with healthy control and CIN, P<0.05. CIN, cervical intraepithelial neoplasia.

A
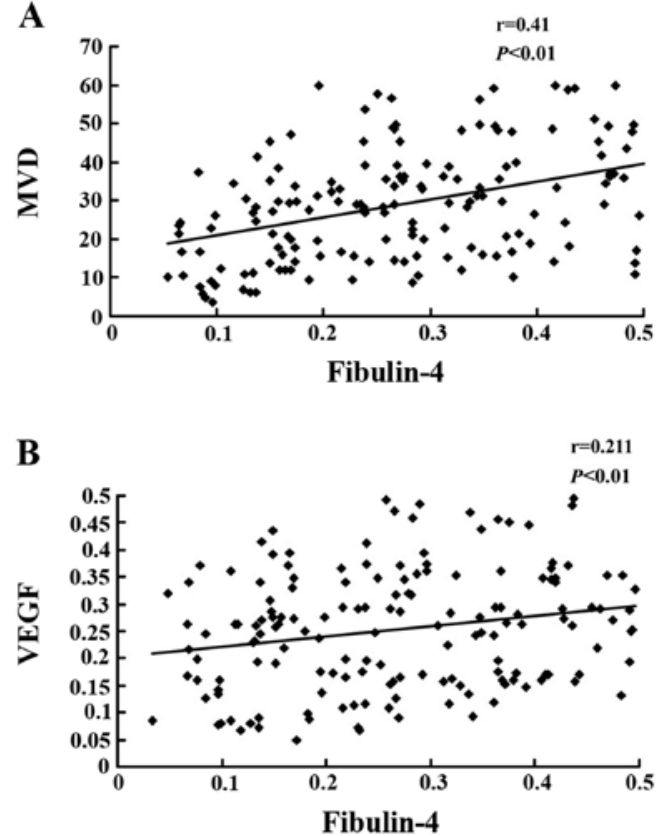

Figure 5. Pearson correlation analysis of fibulin-4 expression and MVD and VEGF. The expression of fibulin-4 was positively correlated with (A) MVD and (B) VEGF.

to be much less than that in the cancer cell cytoplasm, which probably was due to the presence of fewer elastic fibers in cervical tissue. In the basement membranes, however, we found stronger expression of fibulin-4 in CIN when compared with the normal tissue. Real-time RT-PCR experiments confirmed that mRNA expression of fibulin-4 was also upregulated in the cervical carcinoma tissues. Moreover, high fibulin-4 expression was associated with low differentiation, advanced stage and positive lymph node status in the cervical carcinomas. Similar results have been reported in earlier studies on colon cancer; dysregulated expression of the fibulin-4 gene was shown to be associated with human colon tumorigenesis (33). However, contrasting results have also been reported for prostate cancer. By microarray analysis, the fibulin- 4 genes were found to be significantly downregulated in prostate cancer and this result was corroborated by qRT-PCR (22). In this study, fibulin-4 was overexpressed in cervical cancer and was shown to play an important role in tumor development. As is the case for other fibulins, there are controversies in research on fibulin-4; these discrepancies may be attributable to the fact that the tumor microenvironment influences the functions of tumor-associated genes.

Angiogenesis is the process of formation of new microvessels from preexisting vasculature. Once the tumor volume exceeds a few millimeters in diameter, hypoxia and nutrient deprivation trigger tumor cells to exploit their microenvironment by releasing cytokines and growth factors, which then activate normal, quiescent cells around them and initiate a cascade of events resulting in tumor progression. For example, tumor cell-derived VEGF stimulates the sprouting and proliferation of endothelial cells. VEGF is considered the most potent candidate for angiogenesis induction during tumor growth (40). Since angiogenesis is essential for tumor growth and metastasis, controlling tumor-associated angiogenesis is a promising strategy for inhibiting cancer progression. In our study, we sought to determine whether fibulin-4 is associated with angiogenesis. To this end, the Pearson correlation coefficient was calculated to assess the correlation of fibulin-4 with MVD and VEGF expression. We found that fibulin-4 expression was positively correlated with MVD and VEGF expression, which indicated that fibulin-4 may promote angiogenesis. No previous studies concerning fibulin- 4 have reported an association with tumor angiogenesis, although its highly homologous proteins, fibulin-3 and fibulin-5 were found to be associated with tumor angiogenesis. Exogenous and endogenous fibulin-5 were shown to be anti-angiogenic (41). Fibulin-3 was initially found to exert an anti-angiogenic effect (42), but in recent years, several studies have reported that fibulin-3 can promote angiogenesis, particularly in pancreatic adenocarcinoma and cervical cancer. Fibulin-3 gene transfection elevates VEGF expression and MVD (16,17). Since fibulin-4 is highly homologous to fibulin-3 and fibulin-5, we speculate that fibulin-4 may play a significant role in tumor angiogenesis. Pearson correlation tests of MVD and VEGF expression versus the corresponding expression of fibulin- 4 revealed strong direct correlations. Hence, we conclude that fibulin-4 may promote cervical tumor angiogenesis. However, further studies are needed to confirm our speculation, including cell transfection experiments, chorioallantoic membrane assays and tumor xenograft models in nude mice.

High serum levels of fibulin- 4 were found in cervical carcinoma patients when compared with healthy controls and CIN patients, and high fibulin-4 levels were associated with 
low differentiation, advanced stage, and positive lymph node status in cervical carcinomas. This discovery may aid in determining the diagnosis and prognosis of cervical carcinoma. In recent years, fibulins have been recognized as biomarkers for many diseases, such as osteoarthritis, pleural mesothelioma and breast carcinoma. Fibulin-3 and fibulin- 4 may play pathogenic roles in osteoarthritis $(32,43)$. The plasma fibulin-3 and fibulin-1 levels were found to be elevated in patients with mesothelioma and breast carcinoma, respectively $(44,45)$. Novel specific biomarkers can help detect diseases at an earlier stage and tailor treatment strategies for individualized management. Fibulin-4 may be exploited as a tool for the early detection of cervical carcinoma.

In conclusion, fibulin- 4 is a newly identified gene that is overexpressed in cervical carcinoma, promotes angiogenesis, and is associated with poor prognosis. Serum levels of fibulin-4 may be helpful in early diagnosis and determining the prognosis in cases of cervical cancer. Fibulin- 4 may also serve as a novel therapeutic target in patients with cervical carcinoma.

\section{Acknowledgements}

This study was supported by the Independent Innovation Foundation of Shandong University (2012TS088), the National Nature Science Foundation of China (81202056), and the Foundation of Shandong Provincial Science and Technology Development Program (no. 2012G0021820). The funding organizations had no role in the study design, data collection and analysis, decision to publish or preparation of the manuscript.

\section{References}

1. Ferlay J, Shin HR, Bray F, Forman D, Mathers C and Parkin DM: Estimates of worldwide burden of cancer in 2008: GLOBOCAN 2008. Int J Cancer 127: 2893-2917, 2010.

2. Waggoner SE: Cervical cancer. Lancet 361: 2217-2225, 2003.

3. Chen J, Shi D, Liu X, Fang S, Zhang J and Zhao Y: Targeting SPARC by lentivirus-mediated RNA interference inhibits cervical cancer cell growth and metastasis. BMC Cancer 12: 464, 2012.

4. Rao Y, Wang H, Fan L and Chen G: Silencing MTA1 by RNAi reverses adhesion, migration and invasiveness of cervical cancer cells $(\mathrm{SiHa})$ via altered expression of $\mathrm{p} 53$, and $\mathrm{E}$-cadherin $/ \beta$ catenin complex. J Huazhong Univ Sci Technolog Med Sci 31: $1-9,2011$

5. Li Y, Wang W, Wang W, et al: Correlation of TWIST2 up-regulation and epithelial-mesenchymal transition during tumorigenesis and progression of cervical carcinoma. Gynecol Oncol 124: 112-118, 2012

6. de Vega S, Iwamoto T and Yamada Y: Fibulins: multiple roles in matrix structures and tissue functions. Cell Mol Life Sci 66 1890-1902, 2009.

7. Gallagher WM, Currid CA and Whelan LC: Fibulins and cancer: friend or foe? Trends Mol Med 11: 336-340, 2005.

8. Law EW, Cheung AK, Kashuba VI, et al: Anti-angiogenic and tumor-suppressive roles of candidate tumor-suppressor gene, Fibulin-2, in nasopharyngeal carcinoma. Oncogene 31: 728-738, 2012.

9. Yi CH, Smith DJ, West WW and Hollingsworth MA: Loss of fibulin-2 expression is associated with breast cancer progression. Am J Pathol 170: 1535-1545, 2007.

10. Schluterman MK, Chapman SL, Korpanty G, Ozumi K, Fukai T, Yanagisawa $\mathrm{H}$ and Brekken RA: Loss of fibulin-5 binding to betal integrins inhibits tumor growth by increasing the level of ROS. Dis Model Mech 3: 333-342, 2010.

11. Hu Z, Ai Q, Xu H, et al: Fibulin-5 is down-regulated in urothelial carcinoma of bladder and inhibits growth and invasion of human bladder cancer cell line 5637. Urol Oncol 29: 430-435, 2011.
12. Yue W, Sun Q, Landreneau R, Wu C, Siegfried JM, Yu J and Zhang L: Fibulin-5 suppresses lung cancer invasion by inhibiting matrix metalloproteinase-7 expression. Cancer Res 69: 6339-6346, 2009.

13. Moll F, Katsaros D, Lazennec G, et al: Estrogen induction and overexpression of fibulin-1C mRNA in ovarian cancer cells. Oncogene 21: 1097-1107, 2002.

14. Greene LM, Twal WO, Duffy MJ, et al: Elevated expression and altered processing of fibulin-1 protein in human breast cancer. $\mathrm{Br}$ J Cancer 88: 871-878, 2003.

15. Bardin A, Moll F, Margueron R, et al: Transcriptional and posttranscriptional regulation of fibulin- 1 by estrogens leads to differential induction of messenger ribonucleic acid variants in ovarian and breast cancer cells. Endocrinology 146: 760-768, 2005.

16. Seeliger H, Camaj P, Ischenko I, et al: EFEMP1 expression promotes in vivo tumor growth in human pancreatic adenocarcinoma. Mol Cancer Res 7: 189-198, 2009.

17. Song EL, Hou YP, Yu SP, et al: EFEMP1 expression promotes angiogenesis and accelerates the growth of cervical cancer in vivo. Gynecol Oncol 121: 174-180, 2011.

18. Song EL, Chen SG and Wang HQ: The expression of EFEMP1 in cervical carcinoma and its relationship with prognosis. Gynecol Oncol 117: 417-422, 2010.

19. Hu B, Thirtamara-Rajamani KK, Sim H and Viapiano MS: Fibulin-3 is uniquely upregulated in malignant gliomas and promotes tumor cell motility and invasion. Mol Cancer Res 7: 1756-1770, 2009.

20. Kanda M, Nomoto S, Okamura Y, Hayashi M, Hishida M, Fujii T, Nishikawa Y, Sugimoto H, Takeda S and Nakao A: Promoter hypermethylation of fibulin 1 gene is associated with tumor progression in hepatocellular carcinoma. Mol Carcinog 50: 571-579, 2011.

21. Cheng YY, Jin H, Liu X, et al: Fibulin 1 is downregulated through promoter hypermethylation in gastric cancer. Br J Cancer 99: 2083-2087, 2008.

22. Wlazlinski A, Engers R, Hoffmann MJ, et al: Downregulation of several fibulin genes in prostate cancer. Prostate 67: 1770-1780, 2007.

23. Xie L, Palmsten K, MacDonald B, et al: Basement membrane derived fibulin-1 and fibulin-5 function as angiogenesis inhibitors and suppress tumor growth. Exp Biol Med 233: 155-162, 2008.

24. Hwang CF, Chien CY, Huang SC, et al: Fibulin-3 is associated with tumour progression and a poor prognosis in nasopharyngeal carcinomas and inhibits cell migration and invasion via suppressed AKT activity. J Pathol 222: 367-379, 2010.

25. Sadr-Nabavi A, Ramser J, Volkmann J, et al: Decreased expression of angiogenesis antagonist EFEMP1 in sporadic breast cancer is caused by aberrant promoter methylation and points to an impact of EFEMP1 as molecular biomarker. Int J Cancer 124: 1727-1735, 2009.

26. Hu Y, Pioli PD, Siegel E, et al: EFEMP1 suppresses malignant glioma growth and exerts its action within the tumor extracellular compartment. Mol Cancer 10: 123, 2011.

27. Kim EJ, Lee SY, Woo MK, et al: Fibulin-3 promoter methylation alters the invasive behavior of non-small cell lung cancer cell lines via MMP-7 and MMP-2 regulation. Int J Oncol 40: 402-408, 2012.

28. Chen L, Sun B, Zhang S, et al: Influence of microenvironments on microcirculation patterns and tumor invasion-related protein expression in melanoma. Oncol Rep 21: 917-923, 2009.

29. Argraves WS, Greene LM, Cooley MA and Gallagher WM: Fibulins: physiological and disease perspectives. EMBO Rep 4: 1127-1131, 2003.

30. Berk DR, Bentley DD, Bayliss SJ, Lind A and Urban Z: Cutis laxa: a review. J Am Acad Dermatol 66: 842.e1-17, 2012.

31. Huang J, Yamashiro Y, Papke CL, et al: Angiotensin-converting enzyme-induced activation of local angiotensin signaling is required for ascending aortic aneurysms in fibulin-4-deficient mice. Sci Transl Med 5: 183ra58, 1-11, 2013.

32. Xiang Y, Sekine T, Nakamura H, et al: Fibulin- 4 is a target of autoimmunity predominantly in patients with osteoarthritis. J Immunol 176: 3196-3204, 2006.

33. Gallagher WM, Greene LM, Ryan MP, Sierra V, Berger A, Laurent-Puig P and Conseiller E: Human fibulin-4: analysis of its biosynthetic processing and mRNA expression in normal and tumour tissues. FEBS Lett 489: 59-66, 2001. 
34. Ying J, Shan L, Li J, et al: Genome-wide screening for genetic alterations in esophageal cancer by aCGH identifies $11 \mathrm{q} 13$ amplification oncogenes associated with nodal metastasis. PLoS One 7: e39797, 2012.

35. Ormandy CJ, Musgrove EA, Hui R, Daly RJ and Sutherland RL: Cyclin D1, EMS1 and 11q13 amplification in breast cancer. Breast Cancer Res Treat 78: 323-335, 2003.

36. Soumaoro LT, Uetake H, Higuchi T, Takagi Y, Enomoto M and Sugihara K: Cyclooxygenase-2 expression: a significant prognostic indicator for patients with colorectal cancer. Clin Cancer Res 10: 8465-8471, 2004.

37. Weidner N, Folkman J, Pozza F, et al: Tumor angiogenesis: a new significant and independent prognostic indicator in early-stage breast carcinoma. J Natl Cancer Inst 84: 1875-1887, 1992.

38. Landis JR and Koch GG: The measurement of observer agreement for categorical data. Biometrics 33: 159-174, 1977.

39. McLaughlin PJ, Chen Q, Horiguchi M, et al: Targeted disruption of fibulin-4 abolishes elastogenesis and causes perinatal lethality in mice. Mol Cell Biol 26: 1700-1709, 2006.
40. Weis SM and Cheresh DA: Tumor angiogenesis: molecular pathways and therapeutic targets. Nat Med 17: 1359-1370, 2011.

41. Yanagisawa H, Schluterman MK and Brekken RA: Fibulin-5, an integrin-binding matricellular protein: its function in development and disease. J Cell Commun Signal 3: 337-347, 2009.

42. Albig AR, Neil JR and Schiemann WP: Fibulins 3 and 5 antagonize tumor angiogenesis in vivo. Cancer Res 66: 2621-2629, 2006.

43. Henrotin Y, Gharbi M, Mazzucchelli G, Dubuc JE, De Pauw E and Deberg M: Fibulin 3 peptides Fib3-1 and Fib3-2 are potential biomarkers of osteoarthritis. Arthritis Rheum 64: 2260-2267, 2012.

44. Pass HI, Levin SM, Harbut MR, et al: Fibulin-3 as a blood and effusion biomarker for pleural mesothelioma. N Engl J Med 367: 1417-1427, 2012.

45. Piura E and Piura B: Autoantibodies to tumor-associated antigens in breast carcinoma. J Oncol 2010: 264926, 2010. 